\title{
REAL TIME DAMAGE DETECTION THROUGH SINGLE LOW-COST SMART SENSOR
}

\author{
Said Quqa ${ }^{1}$, Luca Landi ${ }^{1}$, and Pier Paolo Diotallevi ${ }^{1}$ \\ ${ }^{1}$ DICAM, University of Bologna \\ Viale del Risorgimento 2, 40136, Bologna, Italy \\ e-mail: \{said.quqa2, 1.landi, pierpaolo.diotallevi\}@unibo.it
}

\begin{abstract}
Continuous vibration-based structural monitoring is increasingly used to assess the state of health of existing structures and infrastructures in environmental conditions through non-invasive methods that allow, in most cases, an early identification of damage. To date, the innovative wireless smart sensor networks are the subject of numerous researches in the field of structural health monitoring, but their use on real structures is still limited, due to problems related to energy consumption and algorithmic optimization. In fact, most of the traditional identification algorithms work in centralized topology and are not suitable for electronic elements with low computational capacity. Nevertheless, the high energy consumption of wireless communication does not allow continuous data transmission for centralized processing in real time. However, the limited costs of new technologies, compared to traditional wired acquisition systems, shifts the interest towards innovative solutions, both from an algorithmic and hardware point of view, in order to provide innovative monitoring systems that can also be used on minor structures, for which traditional systems would be inaccessible. This paper presents a first practical application of a structural identification algorithm specifically designed for lowcost embedded electronic systems on a scaled laboratory model. The proposed solution consists of a single sensing node able to identify natural frequencies in real time, even in conditions of non-stationary excitation and variable structural characteristics. The estimated parameters are periodically uploaded to a cloud platform, where a preliminary real-time damage detection takes place.
\end{abstract}

Keywords: Structural Health Monitoring, Real Time Modal Identification, Damage Detection, Smart Sensors, Teager Energy Operator, Wavelet Packet Transform. 


\section{INTRODUCTION}

In order to ensure the reliability and efficiency of existing civil structures, they have to be properly maintained. Because of the strict constraints of architectural conservation, the preservation of historical buildings mainly means monitoring [1]. For this reason, in recent decades, infrastructures and buildings of strategic and monumental importance have been equipped with advanced vibration-based structural health monitoring systems that allow to detect any changes in the dynamic characteristics potentially connected with ongoing damage [2-3]. Nevertheless, recent news has highlighted the fact that maintenance, and then monitoring, is of the utmost importance also for minor structures and infrastructures, which could undergo fatigue load cycles or other forms of degradation during their rated life time. However, providing each viaduct or building of traditional monitoring systems would be difficult due to the prohibitive costs of traditional sensors and related equipment.

Nowadays, by using the most recent Micro Electro-Mechanical Systems (MEMS) sensors combined with low-cost microcontrollers and wireless transmission modules, it is possible to design innovative monitoring solutions having less impact from the visual and economic point of view with respect to the traditional configurations based on wired piezoelectric sensors [45]. Furthermore, thanks to the computational capacity of the microcontrollers, part of the processing activities can be performed directly on board, making the system particularly suitable for applications in the Internet of Things (IoT) [6-11].

However, these systems have important limitations related with the weak computational abilities of processing units [5]. It is therefore of fundamental importance that the operational algorithms are simple and use few resources, both in terms of memory and energy consumption. For this reason, most of the traditional techniques used for modal identification and damage estimation are not suitable for applications through the innovative low-cost systems.

In this paper, we propose a variant of a structural identification algorithm previously presented for multi-node monitoring networks [12]. This variant is specifically designed for singlenode embedded systems and is able to estimate the first natural frequencies of the monitored structure in real time, in order to notice any changes that could be related with damage.

A first application on a scaled laboratory model is also presented. In particular, two acceleration channels collected by a single triaxial sensor are analyzed in order to compute the instantaneous natural frequencies of the first three vibration modes of the structure. The results are automatically uploaded to the cloud through a platform that allows the real-time visualization of data from any device connected to the Internet. Moreover, a simple procedure for measuring frequency variations with respect to the initial condition is implemented within the online platform in order to perform an early damage detection.

\section{MONITORING SYSTEM}

In this section, a brief description of the hardware and software parts is given. Both these aspects have been designed in order to exploit the computational capabilities of the implemented low-cost electronic devices.

\subsection{Hardware configuration}

The hardware implemented in this study consists of a sensing node and a gateway (Fig. 1). The node is an embedded system composed of a MEMS triaxial accelerometer connected to a microcontroller on which the preliminary signal processing procedures take place through the proposed identification algorithm. In the presented application, the node communicates via a wired one-to-one connection with the gateway, consisting of a single board computer with a 
microprocessor that performs the modal identification. The gateway is wirelessly connected to the Internet in order to upload the identification results to a programmable data-logging application that allows simple post-processing activities, mainly aimed at data representation.

The sensor implemented in the proposed system is the digital low-power and low-noisedensity ADXL-355 triaxial MEMS accelerometer by Analog Devices, with incorporated 20-bit analog-to-digital converter. An amplitude limit of $\pm 2 \mathrm{~g}$ has been selected, corresponding to a sensitivity of $256000 \mathrm{LSB} / \mathrm{g}$ and noise density of $20 \mu \mathrm{g} / \sqrt{\mathrm{Hz}}$ for an output data rate of $500 \mathrm{~Hz}$. The accelerometer is connected via the serial peripheral interface (SPI) to an ARM Cortex-M3 microcontroller unit with $64 \mathrm{kB}$ of flash memory and $20 \mathrm{kB}$ of Random Access Memory (RAM). Some additional LEDs are connected to the microcontroller for debugging purposes.

The sensing node is connected via the Universal Asynchronous Receiver-Transmitter (UART) interface to the gateway, consisting of a Raspberry Pi 3 model B.

The overall cost of the hardware used in this study is very low, and much lower than the cost of traditional piezoelectric sensors, which are usually connected to onerous external monitoring stations.

In order to evaluate the quality of the signal collected through the implemented MEMS sensor, two mono-axial piezoelectric accelerometers 333B40 by PCB Piezotronics have been installed, with a sensitivity of $500 \mathrm{mV} / \mathrm{g}$ and noise density equal to $0.4 \mu \mathrm{g} / \sqrt{\mathrm{Hz}}$ for an output data rate of $1 \mathrm{kHz}$.

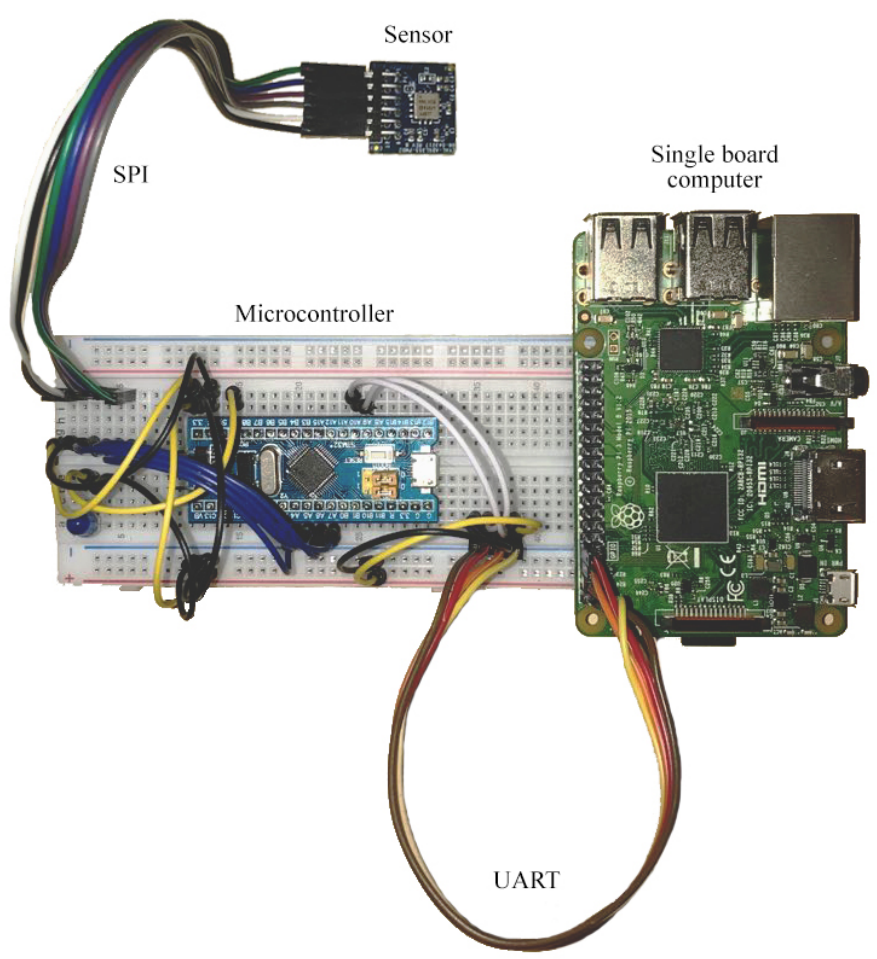

Figure 1: Hardware setup.

\subsection{Algorithm description}

The identification algorithm consists of two steps that are recursively performed to generate a signal-adaptive procedure. The first step involves the construction of a wavelet filter bank used to decompose the acquired signal in separate modal responses, while the second step lies in the real-time estimation of the instantaneous dynamic parameters (frequencies and amplitudes) for each component. 
In particular, the first step involves the preliminary decomposition of the acceleration time histories collected on the structure in different positions and/or directions over a limited time window. The decomposition is performed through a wavelet filter bank, whose properties depend on the parameters selected at the base of the algorithm (i.e., the implemented wavelet function, its order, and the transform level). The signals are therefore decomposed into a series of components, each characterized by a narrow frequency band, which are subsequently grouped into clusters. Then, the signals associated to the same cluster are merged together and only the clusters whose energy (computed as the root mean square of the merged signals) is higher than a user-defined threshold (e.g., the mean value over all the clusters) are selected. Finally, a new filter bank is generated in order to extract by convolution only the selected components. This filter bank is used in the second step of the algorithm, that involves the real-time decomposition of acquired signals and the simultaneous identification of modal parameters.

In the algorithm presented in [12], the clustering procedure is based on the similarity of the operational deflection shapes computed for each component. Since in this application only one sensing node is used, in order to carry out the clustering procedure, the components extracted from two orthogonal channels collected by the same triaxial sensor are analysed, considering a bidirectional element of the operational deflection shape as clustering variable. This method is based on the assumption that the signal window used to build the filter bank is collected under $360^{\circ}$ broadband input excitation.

Moreover, in this application, the modal identification is performed by using the Teager Energy Operator (TEO) [13], that allows to compute the instantaneous frequency and amplitude of each component by analysing extremely reduced signal windows. In particular, by implementing the Discrete-Time Energy Separation Algorithm-1 (DESA-1), only five samples per component are required to estimate an instantaneous parameter value, allowing a real-time and relatively simple evaluation of frequencies and amplitudes, easily performed on board low-cost electronic devices.

In this study, the first step is entirely performed on the gateway, while the second step is carried out partly on the node and partly on the gateway, exploiting the computational capacity of both the devices. The algorithm has been converted into a script for the microprocessor and a firmware for the embedded sensing system, the first aimed at the filter bank construction and modal identification, and the latter used to extract the modal components in real time, as illustrated in Fig. 2.

If the filter bank is no longer suitable for decomposing the modal responses due to a variation of modal parameters, it should be updated. For this reason, each identified frequency value is compared to the limits of the bandpass filters. If these limits are exceeded, the first step of the procedure is repeated.

The natural frequencies are temporarily stored on the on-board memory and, after applying a median filter and a downsampling, are logged on an online platform specifically designed for IoT applications. Here, the variation in terms of frequency for each vibration mode is calculated and plotted in real time, in order to allow users to access the identification results from any location at any time.

In addition, a simple script for damage detection has been written within the online platform. In a "training" window of user-defined duration, the median values of all the identified frequencies are determined. Then, the instantaneous variation of frequency for each mode and an average trend over all the identified modes are computed in the same window with respect to the median values. Finally, the maximum term in the averaged variation is multiplied by a userdefined factor and used as a threshold in damage detection (i.e. as the maximum allowed average frequency variation to consider the structure as undamaged). 


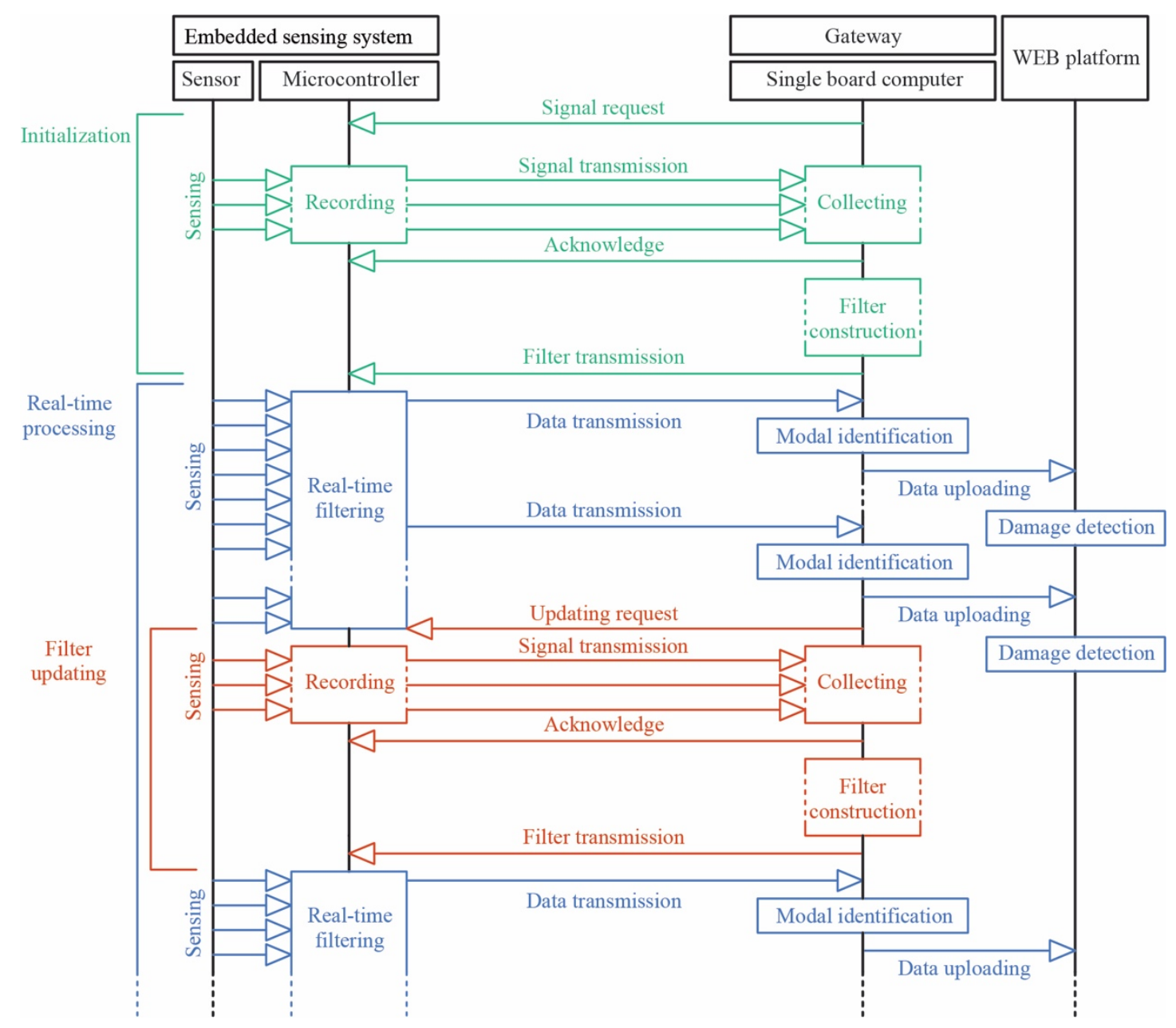

Figure 2: Workflow of the proposed algorithm.

\section{APPLICATION}

As a first application, the proposed monitoring system has been tested on a scaled steel laboratory model. The experimental tests have been conducted to evaluate the reliability of lowcost electronic devices for structural monitoring purposes and the effectiveness of the algorithm in estimating instantaneous natural frequencies.

This section gives a brief description of the specimen and the discussion of the results obtained during the experimental campaign. First, the accelerations recorded through the MEMS sensor implemented in the embedded system are compared with those obtained through a traditional piezoelectric acquisition system during an impulsive test, in order to evaluate the differences in the recorded signals. Subsequently, the results of real-time identification procedure performed through the proposed monitoring system under ambient vibration are reported.

\subsection{Model description and experimental setup}

The model is a three-level steel frame structure (Fig. 3) with a total height of $916 \mathrm{~mm}$, whose base is formed by a $16 \mathrm{~mm}$ thick rectangular plate with main dimensions of 460x300 mm. Each level consists of a $12 \mathrm{~mm}$ thick plate measuring $400 \times 300 \mathrm{~mm}$ and is supported by rectangular columns with a section of $40 \times 3 \mathrm{~mm}$. The connections between columns and plates are formed by L30x3 angle profiles and 72x40x3 mm flat plates held together by couples of M4 bolts with washers, as illustrated in Fig. 4. The net distance between each floor plate is $288 \mathrm{~mm}$. The base plate and the top plate are connected to the columns through stud bolts. 


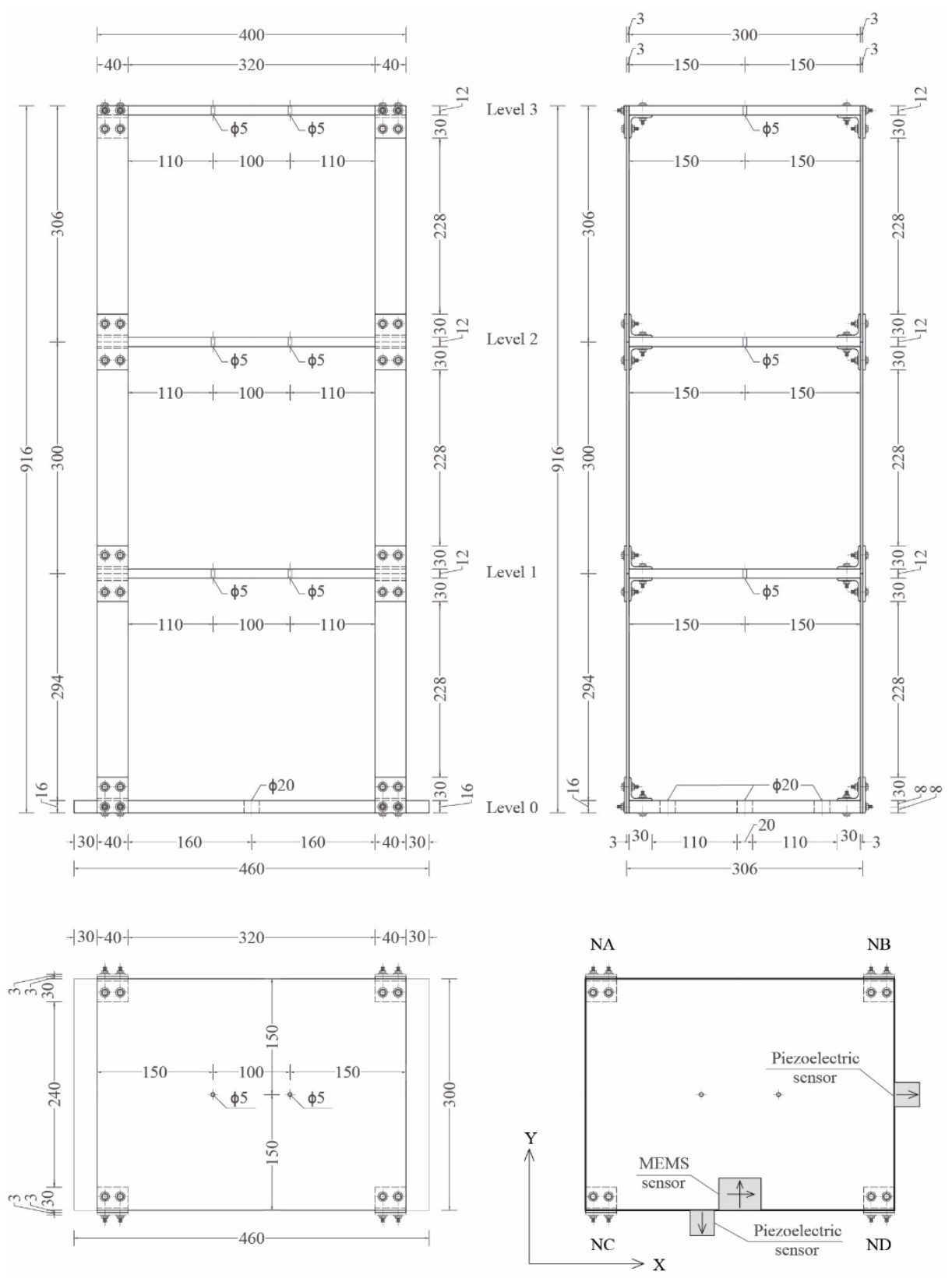

Figure 3: Front, side, and top view of the case study with sensor layout.

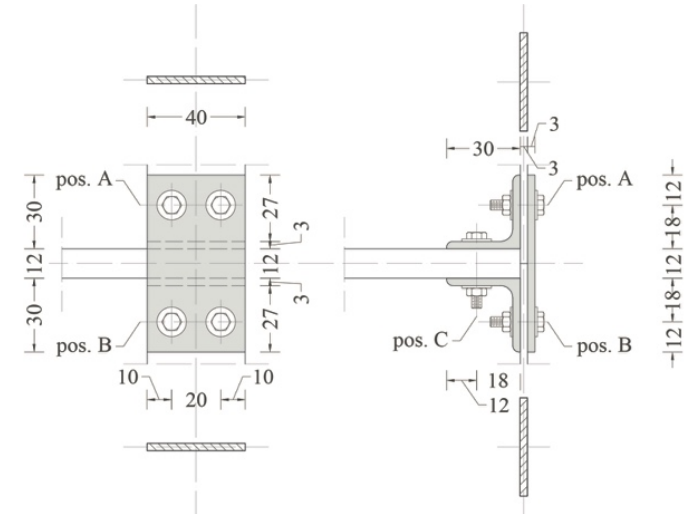

Figure 4: Detail of the connection between columns and plates. 
In a preliminary analysis, the proposed monitoring system and two mono-axial piezoelectric sensors have been mounted on the last level of the structure, as shown in Fig. 3 and 5, in order to collect the response of an impulsive excitation along the same two acceleration channels (i.e., $\mathrm{X}$ and $\mathrm{Y}$ with reference to Fig. 3).

In a second analysis, only the proposed embedded system has been used to monitor the structural conditions under environmental excitation. In this case, several structural configurations (described in Tab. 1) have been reproduced in row, in order to simulate different operative and damaged conditions. After a "training" phase with the reference setup (U, represented in Fig. 3 and 5), four configurations characterized by different mass distributions have been considered. The first three conditions consist of an increase in the floor masses of around $12 \%$ (i.e., $1.5 \mathrm{~kg}$ steel elements placed on the floor plates), at the first level (MA), at the first and second levels (MB), and at all three levels (MC). Then, in condition MD, an additional mass of $1.5 \mathrm{~kg}$ has been introduced at the third level. Subsequently, all the additional masses have been removed in order to bring the structure back to its initial state (R). Then, two damaged configurations have been induced: the first consisted of loosening two bolts in position A for the node NA (with reference to Fig. 3 and 4) at the second level, while in the second configuration two additional bolts in the same position at the first level have been loosened.

\begin{tabular}{lll}
\hline Condition & Description & Duration [s] \\
\hline U & Initial configuration & 585 \\
MA & Additional mass of 12\% at level 1 & 605 \\
MB & Additional masses of 12\% at levels 1 and 2 & 610 \\
MC & Additional masses of 12\% at levels 1,2 and 3 & 550 \\
MD & Additional masses of 12\% at levels 1 and 2, 24\% at level 3 & 605 \\
R & All additional masses removed & 605 \\
DA & Loosened bolts at level 2 & 590 \\
DB & Loosened bolts at levels 1 and 2 & 650 \\
\hline
\end{tabular}

Table 1: Summary of the progressively induced structural conditions.
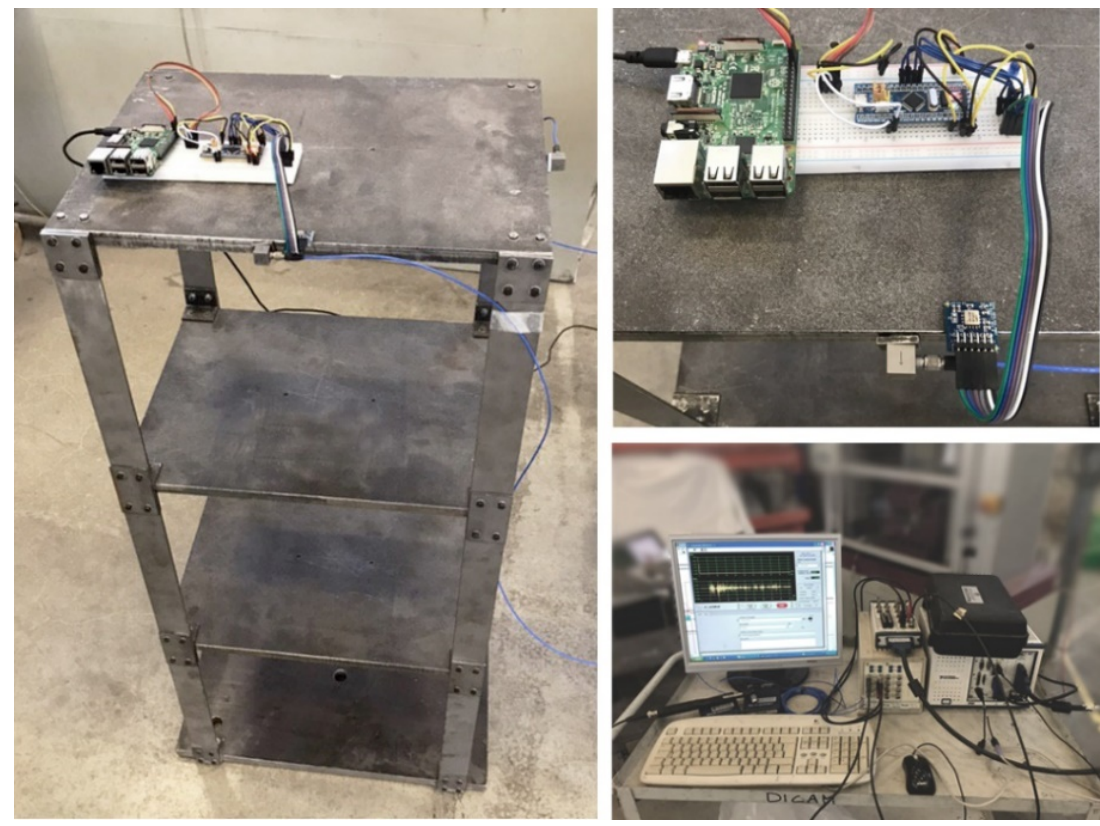

Figure 5: Model equipped with the proposed technology and the traditional sensors (left), detail of the sensor disposition (right-up), and traditional monitoring station used with piezoelectric sensors (right-down). 
Acceleration data on all channels have been collected with a sampling rate of $100 \mathrm{~Hz}$ through the MEMS sensor and $1000 \mathrm{~Hz}$ through the piezoelectric accelerometers. The total duration of the experimental tests of the second analysis is 4800 seconds.

\subsection{Considerations on the signal quality}

The acceleration data collected by piezoelectric sensors have been first compared to those collected using the MEMS sensor. In Fig. 6, the time histories collected along direction Y (with reference to Fig. 3) after an impulsive excitation in the same direction, over a 30-second time window, are reported. Moreover, in Fig. 7 the intervals $0-50 \mathrm{~Hz}$ of the Fourier spectra of these signals are represented.

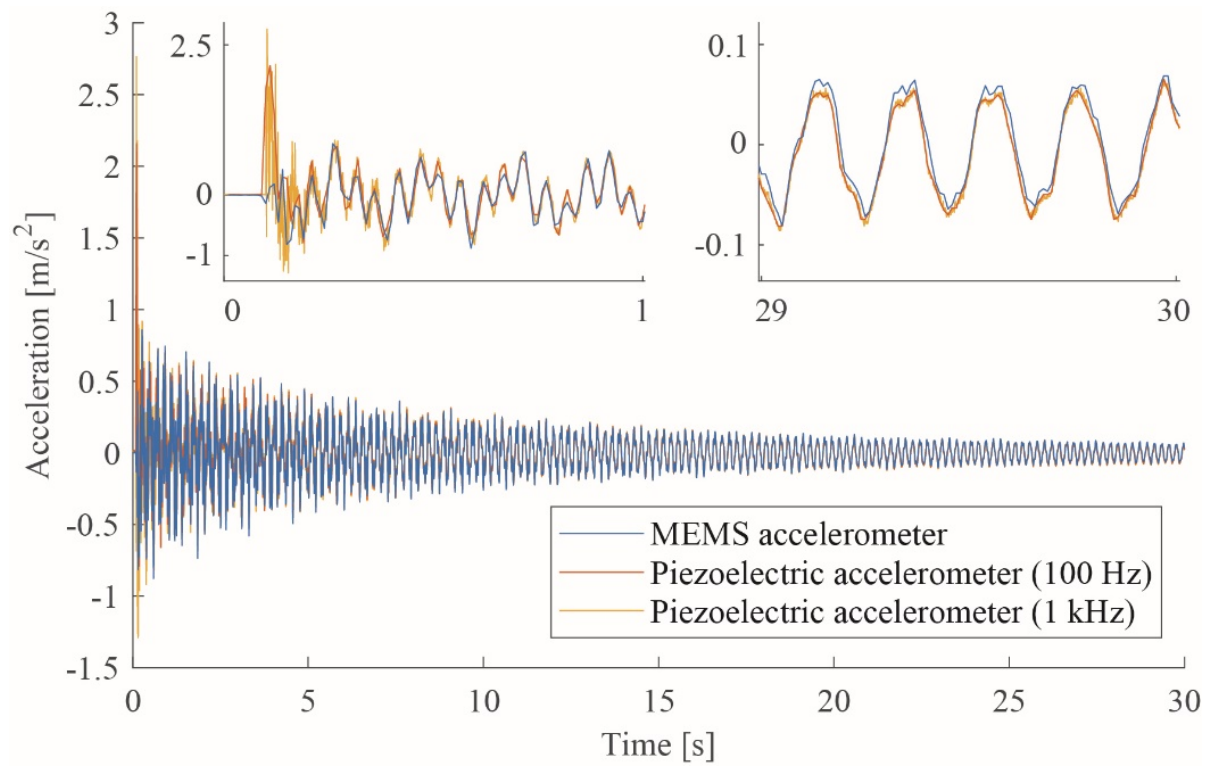

Figure 6: Comparison between the acceleration impulse responses in Y direction collected by the piezoelectric and MEMS accelerometers. In the upper part of the figure, two zoomed windows of 1 second are reported.

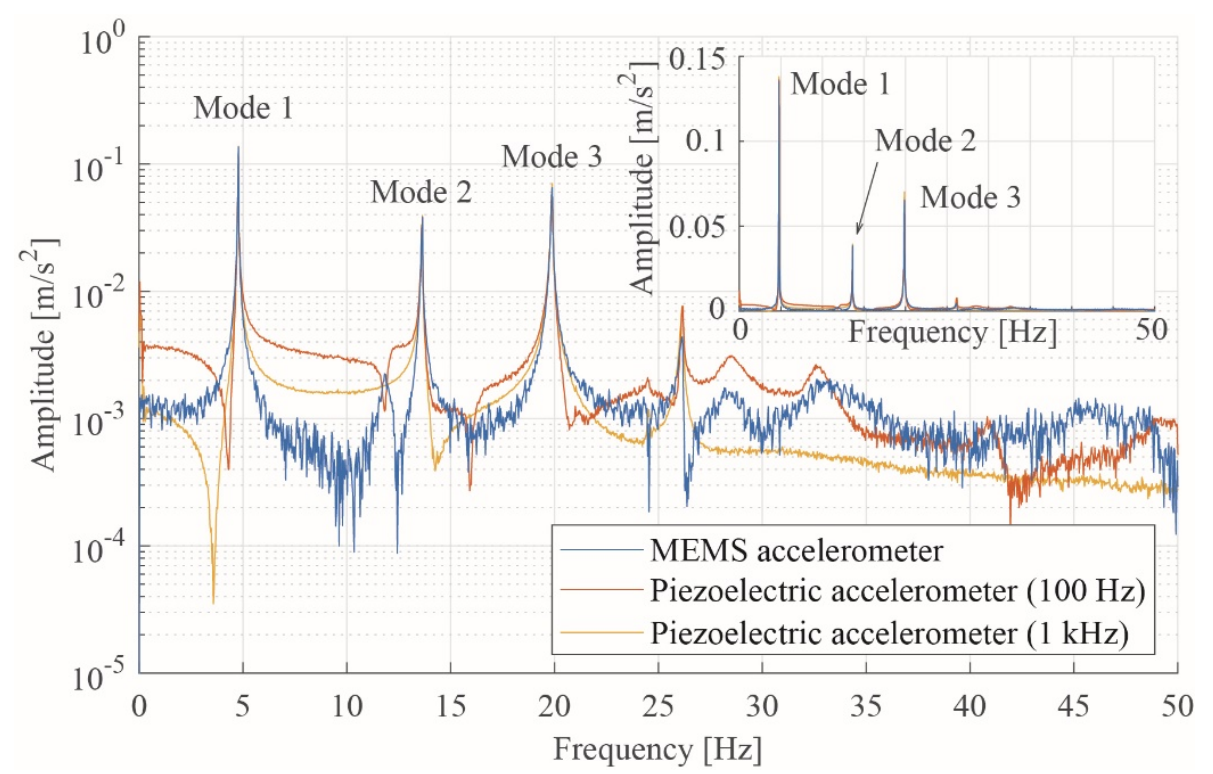

Figure 7: Fourier spectra obtained from the impulse responses. The main figure is represented on logarithmic scale, while the upper graph is on linear scale. 
In particular, the first signal is the acceleration collected by the MEMS sensor, which is compared to the second signal obtained through the piezoelectric accelerometer, downsampled by a factor 10 in order to have the same sampling rate as the first. The third signal is the original acceleration recorded by the piezoelectric sensor, reported as a reference.

From Fig. 6 it is possible to observe, especially in the first part of the recording, how the signal obtained by using the piezoelectric sensor is considerably more accurate than the one measured through the MEMS sensor, also for the downsampled version. In fact, the ratio between the root mean square (RMS) of the difference between the two signals (considering the downsampled version for the piezoelectric sensor) and the RMS of the signal obtained through the MEMS sensor is equal to 0.42. However, it is possible to observe how the upper parts of first three peaks in the frequency spectra reported in Fig. 7, are almost coincident. In particular, the main differences are for lower amplitudes, and noticeable only by representing the spectrum on a logarithmic scale. For this reason, even if the MEMS sensor is less accurate, it still allows to correctly identify the first natural frequencies. The three identified peaks also correspond to the natural frequencies associated with the first three translational vibration modes in the $\mathrm{Y}$ direction of a finite element model designed to confirm the results obtained from modal analysis.

\subsection{Results of the proposed system}

Following the analyses performed to evaluate the signal quality, the modal identification algorithm has been applied using only the MEMS sensor under environmental vibration.

The wavelet function Fejér-Korovkin 14 has been chosen to build the filter bank used in the first phase of the identification algorithm. The wavelet transform has been performed up to the fifth order, generating a bank of 32 bandpass filters. Following the clustering procedure, based on the parameters computed over a 10-seconds initialization window, three signal components have been selected, associated with the first three modal responses.

With the application of DESA-1 algorithm, each value of the instantaneous natural frequencies has been computed considering only 5 acceleration samples. In order to lighten the computed parameters, a median value of the frequency has been calculated every 5 seconds. The results obtained for each vibration mode have been then uploaded to the cloud platform. The instantaneous frequency for each identified mode is represented over time in Fig. 8 as a solid line, while the state representing the reference condition, computed as the median value of each natural frequency over a training window of 50 samples obtained during the state $U$, is reported as dotted line.

During the real-time processing procedure, the estimated frequencies exceeded the limits of bandpass filters in two instants, indicated as vertical violet dashed lines in Fig. 8, where the filter bank has been updated following the scheme of Fig. 2. It can be noted that the proposed algorithm has shown particularly suitable also for detecting sudden changes in modal parameters, since the results reported in Fig. 8 has been calculated continuously, without interrupting the identification procedure during the changes in structural conditions.

Within the cloud platform, a script has been written to evaluate the frequency variation with respect to the baseline, in order to indicate any possible state of damage. The frequency variation has been evaluated with respect to the reference value computed during the training window. The results for the three identified modes and an average trend are reported as functions of time in Fig. 9. In this application, the criterion selected to define a condition as probably damaged consists in evaluating whether the average instantaneous frequency variation exceeds more than twice the maximum average variation value encountered in the training window. 


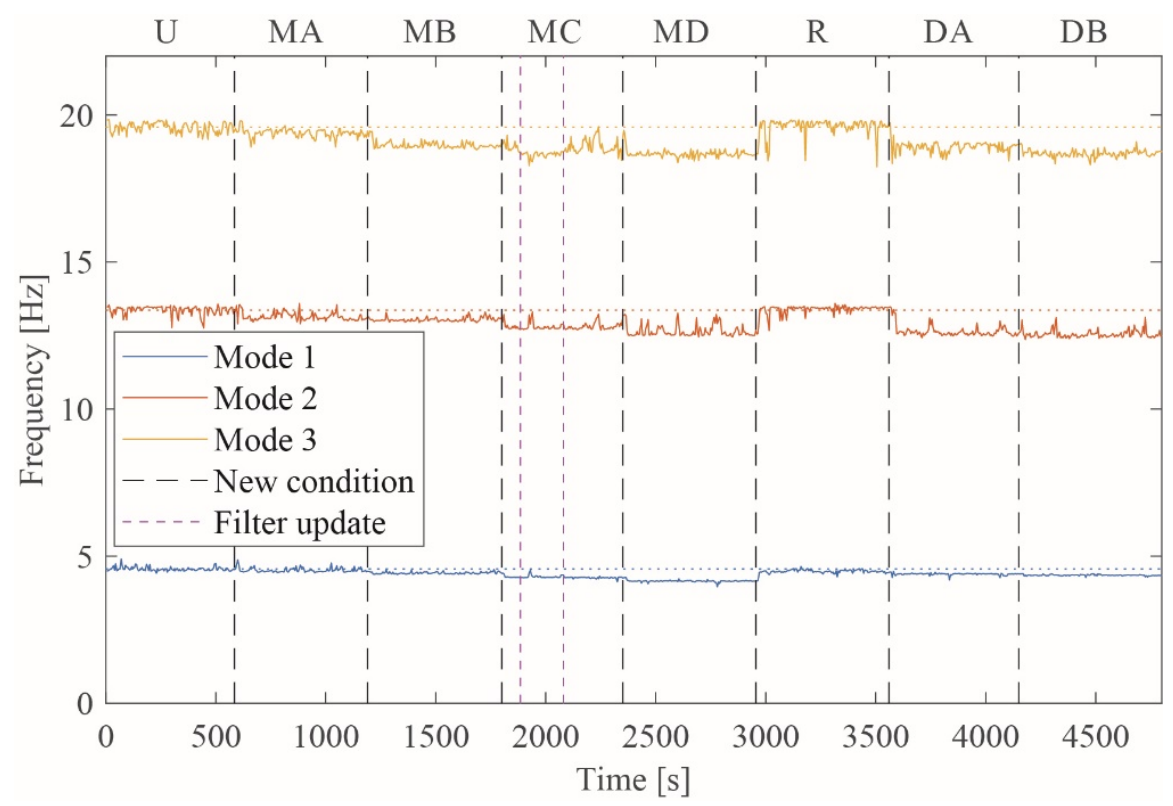

Figure 8: Instantaneous frequencies estimated through the proposed system during the experimental tests.

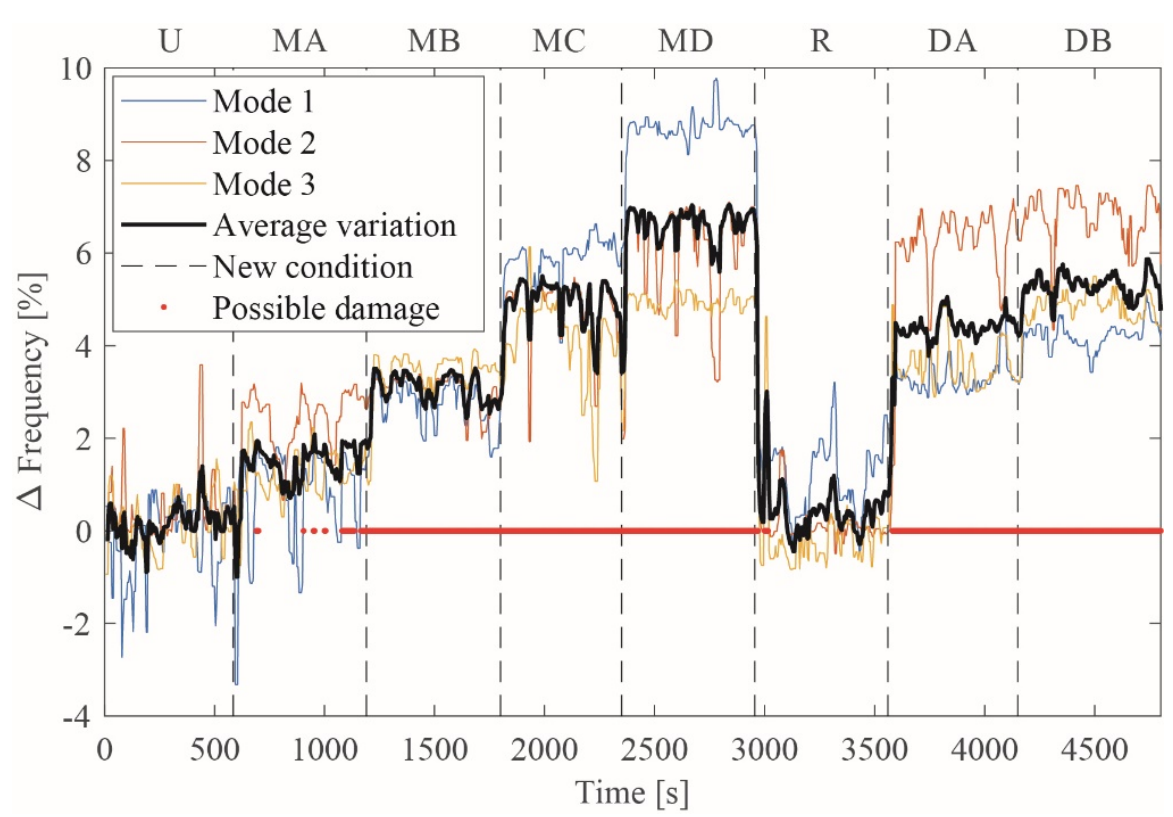

Figure 9: Instantaneous variation of frequency and damage detection performed through the algorithm written within the online platform.

In Fig. 9, the red dots represented on the time axis indicate the instantaneous detection of a "possibly damaged" configuration. Where the red sign appears continuous, a persistent damaged condition is detected, in which the frequency variation does not return within the bounds evaluated during the training interval. It is observable how all the operational and damaged condition have been indicated as "possibly damaged", with some missing alarms for the MA condition, where the frequency variation is in some parts comparable with the values of the training window. However, by applying a median filter with a window of 10 samples to the instantaneous frequency variations, the whole condition MA would be referred to as "possibly damaged" and the detection would become more robust. 


\section{CONCLUSIONS}

In this paper, the implementation of a modal identification and damage detection algorithm on a specifically-designed electronic monitoring system has been presented. The algorithm proved to be particularly effective for the identification of dynamic parameters of a scale laboratory model, even if the quality of the accelerations obtained through the implemented MEMS sensor is lower than the quality of the recordings collected by piezoelectric accelerometers. In fact, also small variations in natural frequencies have been evaluated with sufficient accuracy to allow the distinction between all the considered structural configurations.

On-board processing was made possible by the simplicity and low amount of memory required by the identification algorithm. This allows to apply the proposed method through small, non-invasive and low-cost smart devices.

\section{REFERENCES}

[1] ICOMOS charter (2003) Principles for the Analysis, Conservation and Structural Restoration of Architectural Heritage. Ratified by the ICOMOS 14th General Assembly, in Victoria Falls, Zimbabwe, October 2003.

[2] A. Saisi, C. Gentile, A. Ruccolo, Continuous monitoring of a challenging heritage tower in Monza, Italy. Journal of Civil Structural Health Monitoring, 8(1), 77-90, 2017.

[3] Z. Chen, X. Zhou, X. Wang, L. Dong, Y. Qian, Deployment of a smart structural health monitoring system for long-span arch bridges: a review and a case study. Sensors, 17(9), 2151, 2017.

[4] A.B. Noel, A. Abdaoui, T. Elfouly, M.H. Ahmen, A. Badawy, M.S. Shehata, Structural health monitoring using wireless sensor networks: a comprehensive survey. IEEE Communications Surveys \& Tutorials, 19(3), 1403-1423, 2017.

[5] J.P. Lynch, K.J. Loh, A summary review of wireless sensors and sensor networks for structural health monitoring. The Shock and Vibration Digest, 38(2), 91-128, 2006.

[6] H. Jo, S.-H. Sim, T. Nagayama, Development and application of high-sensitivity wireless smart sensors for decentralized stochastic modal identification. Journal of Engineering Mechanics, 138(6), 683-694, 2012.

[7] Y. Gao, B.F. Spencer Jr, M. Ruiz-Sandoval, Distributed computing strategy for structural health monitoring. Structural Control and Health Monitoring, 13, 488-507, 2006.

[8] A.T. Zimmerman, M. Shiraishi, R.A. Swartz, J.P. Lynch, Automated modal parameter estimation by parallel processing within wireless monitoring systems. Journal of infrastructure systems, 14(1), 102-113, 2008.

[9] S.-H. Sim, J.F. Carbonell-Márquez, B.F. Spencer Jr, H. Jo, Decentralized random decrement technique for efficient data aggregation and system identification in wireless smart sensor networks. Probabilistic Engineering Mechanics, 26, 81-91, 2011.

[10] A. Sadhu, S. Narasimhan, A decentralized blind source separation algorithm for ambient modal identification in the presence of narrowband disturbances. Structural Control and Health Monitoring, 21, 282-302, 2014. 
[11] S. Jeong, Y. Zhang, R. Hou, J.P. Lynch, H. Sohn, K.H. Law, A cloud-based information repository for bridge monitoring applications. J.P. Lynch ed. Proceedings of the SPIE Smart Structures/NDE Conference, Baltimore, Maryland, USA, 2016.

[12] S. Quqa, L. Landi, P.P. Diotallevi, Vibration-based structural damage detection using a decentralized network with limited sensors. $16^{\text {th }}$ European Conference on Earthquake Engineering (16ECEE), Thessaloniki, Greece, 2018.

[13] P. Maragos, J.F. Kaiser, T.F. Quartieri, Energy separation in signal modulations with application to speech analysis. IEEE Transactions on Signal Processing, 41(10), 3024-3051, 1993. 